\title{
Paraneoplastic Opsoclonus-myoclonus Syndrome with Anti- Hu and Anti-SOX-1 Antibodies after Immune-checkpoint Inhibitor Treatment Combined with Chemotherapy in a Patient with Small-cell Lung Cancer
}

Hironori Arai ${ }^{1}$, Yoshikazu Utsu ${ }^{1}$, Joji Horio ${ }^{1}$, Shogo Furukawa $^{2}$ and Yuriko Kikkawa ${ }^{2}$

\begin{abstract}
:
A 69-year-old man with advanced small-cell lung cancer achieved partial remission after 3 courses of immunochemotherapy that included atezolizumab. Ten days after the last treatment, he developed paraneoplastic opsoclonus-myoclonus syndrome and required mechanical ventilation. Serology testing detected anti-Hu and anti-SOX-1 antibodies. Despite steroid pulse therapy, various anticonvulsants, continuous intravenous sedation, and a fourth course of chemotherapy without atezolizumab, his condition failed to improve. Paraneoplastic opsoclonus-myoclonus syndrome with autoantibodies after immune-checkpoint inhibitor treatment has not been reported previously. Although a causal relationship between immune-checkpoint inhibitors and paraneoplastic syndromes has been suggested, the mechanism remains unknown.
\end{abstract}

Key words: opsoclonus-myoclonus syndrome, paraneoplastic syndrome, immune checkpoint inhibitor, smallcell lung carcinoma

(Intern Med 61: 71-74, 2022)

(DOI: 10.2169/internalmedicine.7167-21)

\section{Introduction}

Paraneoplastic neurological syndromes are relatively rare, occurring in fewer than $1 \%$ of carcinoma patients, and are considered a type of autoimmune disease in which a tumor or nerve tissue provides the antigen. The central nervous system, peripheral nerves, neuromuscular junction, and muscles are all potential immune targets. Neurological symptoms usually precede tumor detection and then progress subacutely to determine the type of clinical syndrome. Characteristic autoantibodies are often detected in the serum or cerebrospinal fluid (CSF) of affected patients. Paraneoplastic opsoclonus-myoclonus syndrome (POMS) is an extremely rare paraneoplastic syndrome that presents with opsoclonus, cerebellar ataxia, and myoclonus of the trunk and limbs. Although reports of paraneoplastic syndromes after the administration of immune-checkpoint inhibitors (ICIs) have been increasing, POMS with autoantibodies has not been reported previously.

\section{Case Report}

A 69-year-old man with a history of bipolar disorder was referred to our hospital with a $7.2-\mathrm{cm}$-diameter mass in the posterior mediastinum and small right pleural effusion. After a detailed examination, he was diagnosed with advanced stage IV small-cell lung cancer. No bone or brain metastases were found. His Eastern Cooperative Oncology Group Performance Status was 0.

The patient was treated with combined chemotherapy consisting of carboplatin at an area under the concentrationtime curve of 6 , atezolizumab $1,200 \mathrm{mg}$ on day 1 , and etoposide $100 \mathrm{mg} / \mathrm{m}^{2}$ on days $1-3$ every 3 weeks for 3 courses, which resulted in partial remission (Fig. 1). His pro-gastrin-releasing peptide levels decreased markedly from $2,150 \mathrm{pg} / \mathrm{mL}$ to $22.8 \mathrm{pg} / \mathrm{mL}$, and his neuron-specific enolase levels decreased from $18.8 \mathrm{ng} / \mathrm{mL}$ to $8.2 \mathrm{ng} / \mathrm{mL}$. Ten days

${ }^{1}$ Department of Hematology and Oncology, Japanese Red Cross Narita Hospital, Japan and ${ }^{2}$ Department of Neurology, Japanese Red Cross Narita Hospital, Japan

Received for publication January 26, 2021; Accepted for publication May 10, 2021

Correspondence to Dr. Hironori Arai, h.arai@naritasekijyuji.jp. 
(a)

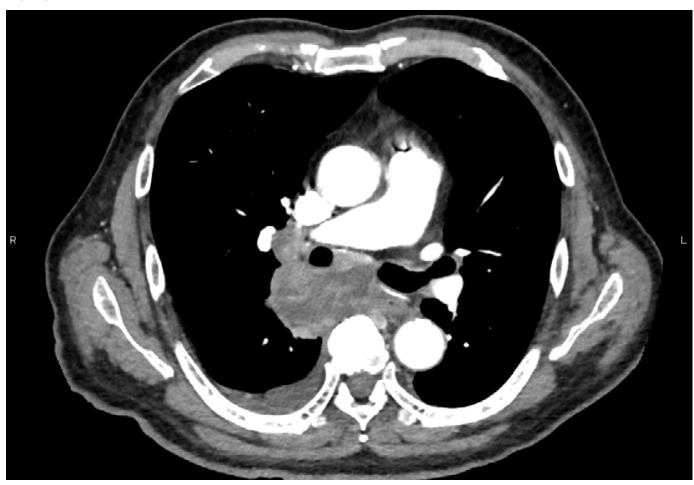

(b)

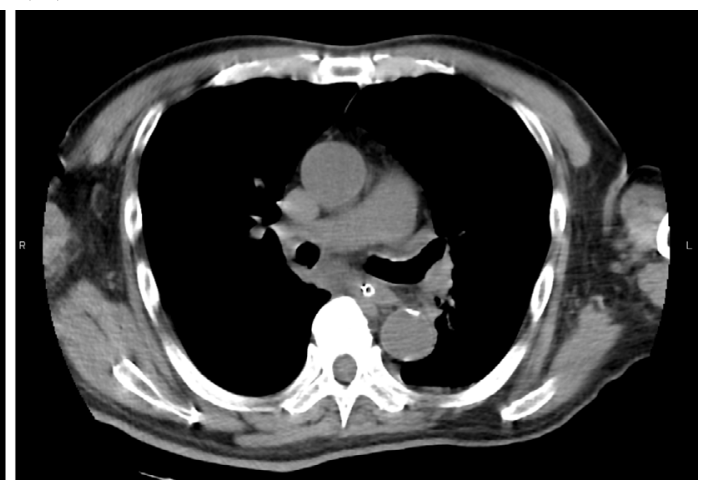

Figure 1. Radiologic appearance of the tumor on computed tomography. (a) Before treatment, a 7.2-cm-diameter mass and right pleural effusion were detected in the posterior mediastinum. (b) Most of the tumor and pleural effusion disappeared after three courses of immunochemotherapy.
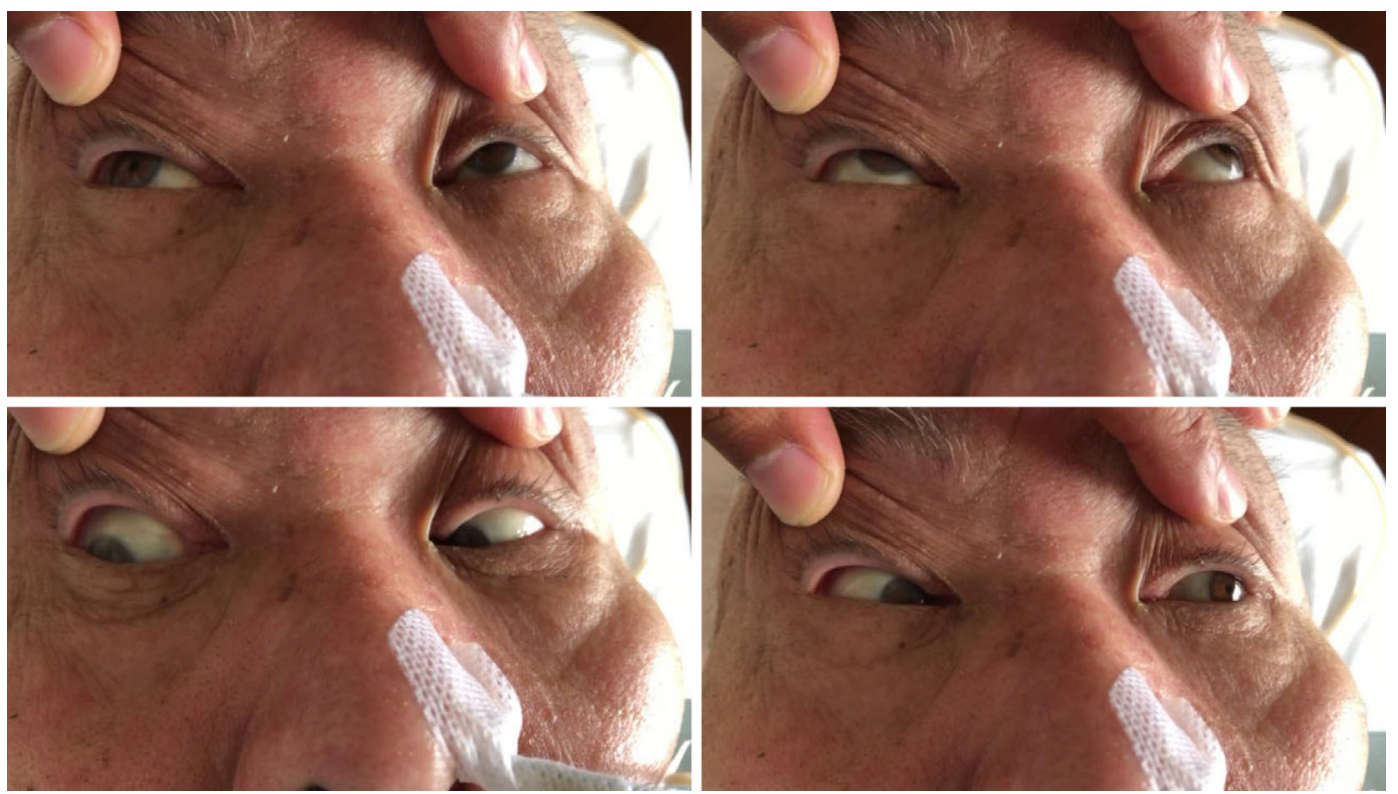

Figure 2. Opsoclonus. The patient exhibited unpredictable, rapid, involuntary, and multivectorial conjugate eye movements without intersaccadic intervals.

after the last treatment, he was admitted to the hospital because of vomiting, anorexia, fatigue, dyspnea, and tremor in both upper limbs. Limb myoclonus, dysarthria, and loss of consciousness occurred the next day, followed by opsoclonus 5 days later (Fig. 2). A high dose of diazepam was administered to stop the tremor, but it had little effect and caused respiratory arrest. The patient was intubated and received continuous midazolam, levetiracetam, lacosamide, perampanel, and clonazepam, which did not control the myoclonus. A CSF examination showed mild lymphocytic pleocytosis (17 cells $/ \mu \mathrm{L})$, mildly increased protein $(71 \mathrm{mg} /$ $\mathrm{dL}$ ), and an IgG index of 0.73 ; CSF cytology and viral marker testing were negative. Autoantibodies were not examined in the CSF. Computed tomography of the head, contrast-enhanced brain magnetic resonance imaging, and blood testing showed no obvious abnormalities. Serological tests were strongly positive for anti-Hu and anti-SOX-1 anti- bodies.

The patient was diagnosed with POMS and received three courses of steroid pulse therapy with no effect. Radical treatment with lung cancer chemotherapy was considered necessary. Carboplatin plus etoposide therapy without atezolizumab was started on the 20th day of mechanical ventilation without response. Despite chemotherapy and deep sedation, the patient's opsoclonus and myoclonus persisted. Because the patient continued to require mechanical ventilation, tracheotomy was performed, and he was transferred to a long-term care facility with the use of a ventilator.

\section{Discussion}

Based on the guidelines proposed by the Joint Task Force of the European Federation of Neurological Societies and the Peripheral Nerve Society, our patient was diagnosed 
with POMS with autoantibodies. Although reports of paraneoplastic syndromes occurring after the administration of ICIs have been increasing, given their widespread use (1-8), POMS with autoantibodies has not been reported previously $(9,10)$.

Paraneoplastic syndromes usually precede the detection of cancer, and the symptoms persist in many cases after treatment. No case reports showing the occurrence of paraneoplastic syndrome during successful chemotherapy without ICI have been published, which suggests that POMS in this case was caused by an ICI. Paraneoplastic syndromes can be characterized into two main categories based on the antigenic sites involved. In the first category, cell surface receptors/ion channels are involved, and early plasma exchange for antibody removal, high-dose immunoglobulin therapy, and steroid therapy are effective for improving neurological symptoms $(8,11)$. In the second category, proteins in the cytoplasm and nucleus are involved in a cytotoxic $\mathrm{T}$ lymphocyte-mediated manner, and treatment is mostly ineffective $(8,11)$. Paraneoplastic syndromes with antibodies fall into the second category (1).

Anti-Hu and anti-SOX-1 antibodies are commonly observed in small-cell lung cancer. Anti-SOX-1 antibodies are positive in approximately $30 \%$ of paraneoplastic syndrome cases with anti-Hu antibodies, but the significance of both antibodies remains unclear (12). In addition, approximately $40 \%$ of all small-cell lung cancers are positive for anti-SOX1 antibodies, regardless of the presence of paraneoplastic syndrome. Therefore, although anti-Hu and anti-SOX-1 antibodies can be used as biomarkers of paraneoplastic syndromes, there is no evidence to date that they are pathogenic. Furthermore, these antibodies were reported to not directly affect the survival of small-cell lung cancer patients $(12,13)$.

Cases of opsoclonus-myoclonus syndrome associated with pediatric neuroblastoma or autoimmune disease can benefit from immunosuppressive therapy, such as rituximab (14); however, adult-onset POMS is generally considered resistant (15). Considering our patient's age and serology findings, a poor treatment response was expected. Although the patient was intubated for respiratory arrest caused mainly by diazepam, the fact that his respiratory condition did not improve suggests that other paraneoplastic syndromes might have occurred. However, we were unable to examine the patient further because of his poor general condition.

In accordance with the American Society of Clinical Oncology guidelines for the treatment of immune-related adverse events, we administered high-dose steroid therapy after discontinuing atezolizumab, with little effect (16). The main treatment for paraneoplastic syndromes is treatment of the primary disease. However, Manson et al. reported no significant correlation between tumor reduction and syndrome improvement in patients with ICI-associated paraneoplastic syndrome (17). This suggests that treatment of the cancer itself may not be effective. Our patient developed POMS despite tumor reduction with chemotherapy. Furthermore, the subsequent resumption of chemotherapy did not result in any improvement in his POMS. The onset or exacerbation of paraneoplastic syndromes can occur within a few months after ICI treatment initiation $(17,18)$, as illustrated in our patient. Although whether or not there is a causal relationship between ICIs and paraneoplastic syndromes remains unclear, autoantibodies may be a biomarker for the development of ICI-associated paraneoplastic syndromes $(7,18)$. Thus far, no treatment for ICI-associated paraneoplastic syndromes has been established. Natalizumab, an anti- $\alpha 4 \beta 1$ integrin monoclonal antibody used for the treatment of multiple sclerosis patients, has been reported to be effective (19). Natalizumab prevents lymphocyte entry at the blood-brain barrier and does not interfere with anti-tumor ICI effects. Therefore, the further investigation of natalizumab as a treatment for paraneoplastic syndromes is warranted (3).

\section{Conclusion}

Paraneoplastic syndromes are serious complications that often result in a poor outcome. The incidence of ICIassociated paraneoplastic syndromes has been increasing, but their pathogenesis remains unclear. Therefore, caution when using ICIs and careful management of paraneoplastic syndromes in small-cell lung cancer patients are crucial to ensure good outcomes. Further studies of the relationship between ICIs and paraneoplastic syndromes are needed.

The authors declare that the procedures followed were in accordance with the Declaration of Helsinki and that human investigations were performed after approval by a relevant institutional review board.

\section{The authors state that they have no Conflict of Interest (COI).}

\section{Acknowledgement}

We thank J. Ludovic Croxford, $\mathrm{PhD}$, for editing a draft of this manuscript.

\section{References}

1. Papadopoulos KP, Romero RS, Gonzalez G, Dix JE, Lowy I, Fury M. Anti-Hu-associated autoimmune limbic encephalitis in a patient with PD-1 inhibitor-responsive myxoid chondrosarcoma. Oncologist 23: 118-120, 2018.

2. Hardy-Werbin M, Arpi O, Taus A, et al. Assessment of neuronal autoantibodies in patients with small-cell lung cancer treated with chemotherapy with or without ipilimumab. Oncoimmunology 7: e $1395125,2018$.

3. Gill A, Perez MA, Perrone CM, Bae CJ, Pruitt AA, Lancaster E. A case series of PD-1 inhibitor-associated paraneoplastic neurologic syndromes. J Neuroimmunol 334: 576980, 2019.

4. Vogrig A, Muniz-Castrillo S, Joubert B, et al. Central nervous system complications associated with immune checkpoint inhibitors. J Neurol Neurosurg Psychiatry 91: 772-778, 2020.

5. Appelbaum J, Wells D, Hiatt JB, et al. Fatal enteric plexus neuropathy after one dose of ipilimumab plus nivolumab: a case report. J Immunother Cancer 6: 82, 2018.

6. Vogrig A, Fouret M, Joubert B, et al. Increased frequency of anti$\mathrm{Ma} 2$ encephalitis associated with immune checkpoint inhibitors. Neurol Neuroimmunol Neuroinflamm 6: e604, 2019. 
7. Matsuoka H, Kimura H, Koba H, et al. Nivolumab-induced limbic encephalitis with anti-Hu antibody in a patient with advanced pleomorphic carcinoma of the lung. Clin Lung Cancer 19: e597-e 599, 2018.

8. Graus F, Dalmau J. Paraneoplastic neurological syndromes in the era of immune-checkpoint inhibitors. Nat Rev Clin Oncol 16: 535548, 2019.

9. Vedeler CA, Antoine JC, Giometto B, et al. Management of paraneoplastic neurological syndromes: report of an EFNS Task Force. Eur J Neurol 13: 682-690, 2006.

10. Graus F, Delattre JY, Antoine JC, et al. Recommended diagnostic criteria for paraneoplastic neurological syndromes. J Neurol Neurosurg Psychiatry 75: 1135-1140, 2004.

11. Tanaka K. Paraneoplastic neurological syndromes with reference to anti-neuronal autoantibodies. Rinsho Shinkeigaku 50: 371-378, 2010 (In Japanese, Abstract in English).

12. Stich O, Klages E, Bischler P, et al. SOX1 antibodies in sera from patients with paraneoplastic neurological syndromes. Acta Neurol Scand 125: 326-331, 2012.

13. Titulaer MJ, Klooster R, Potman M, et al. SOX antibodies in small-cell lung cancer and Lambert-Eaton myasthenic syndrome: frequency and relation with survival. J Clin Oncol 27: 4260-4267, 2009.

14. Pranzatelli MR, Tate ED, Travelstead AL, et al. Rituximab (antiCD20) adjunctive therapy for opsoclonus-myoclonus syndrome. J Pediatr Hematol Oncol 28: 585-593, 2006.
15. Bataller L, Graus F, Saiz A, Vilchez JJ; Spanish OpsoclonusMyoclonus Study Group. Clinical outcome in adult onset idiopathic or paraneoplastic opsoclonus-myoclonus. Brain 124: 437443, 2001.

16. Brahmer JR, Lacchetti C, Schneider BJ, et al. Management of immune-related adverse events in patients treated with immune checkpoint inhibitor therapy: American Society of Clinical Oncology Clinical Practice Guideline. J Clin Oncol 36: 1714-1768, 2018.

17. Manson G, Maria ATJ, Poizeau F, et al. Worsening and newly diagnosed paraneoplastic syndromes following anti-PD-1 or anti-PDL1 immunotherapies, a descriptive study. J Immunother Cancer 7: 337, 2019.

18. Vogrig A, Muniz-Castrillo S, Desestret V, Joubert B, Honnorat J. Pathophysiology of paraneoplastic and autoimmune encephalitis: genes, infections, and checkpoint inhibitors. Ther Adv Neurol Disord 13: 1756286420932797, 2020.

19. Hottinger AF, de Micheli R, Guido V, Karampera A, Hagmann P, Du Pasquier R. Natalizumab may control immune checkpoint inhibitor-induced limbic encephalitis. Neurol Neuroimmunol Neuroinflamm 5: e439, 2018.

The Internal Medicine is an Open Access journal distributed under the Creative Commons Attribution-NonCommercial-NoDerivatives 4.0 International License. To view the details of this license, please visit (https://creativecommons.org/licenses/ by-nc-nd/4.0/).

(C) 2022 The Japanese Society of Internal Medicine Intern Med 61: 71-74, 2022 